\title{
VARIABLES SOCIOEMOCIONALES Y DE ATENCIÓN PLENA EN EL BIENESTAR SUBJETIVO
}

\author{
Isabel Luján Henríquez \\ Universidad de Las Palmas de Gran Canaria (ULPGC) \\ isabel.lujan@ulpgc.es \\ Rosa Delia Martín Rodríguez \\ Universidad Internacional de la Rioja (UNIR) \\ rosa.martin@unir.net
}

Recepción Artículo: 17 octubre 2021 Admisión Evaluación: 17 octubre 2021 Informe Evaluador 1: 18 octubre 2021 Informe Evaluador 2: 19 octubre 2021 Aprobación Publicación: 20 octubre 2021

\section{RESUMEN}

En las últimas décadas ha cobrado especial interés el estudio, tanto teórico como empírico, sobre la valoración que realizan las personas en relación a la satisfacción que pueden sentir acerca de su vida con la finalidad de descubrir, prevenir y promover las variables y los recursos que inciden para poder tener una vida plena y feliz. En esta línea, desde la perspectiva de la Psicología Positiva, se planteó una investigación con dos objetivos fundamentales. Por un lado, conocer qué perfil socioemocional (Moldes Mentales, Pensamiento Constructivo e Inteligencia emocional) subyace en las personas que han participado en un programa de Atención Plena y si este tipo de variables permiten predecir el índice de bienestar subjetivo. Por otro lado, valorar la repercusión de la participación en un programa Mindfulness (atención plena) sobre el bienestar subjetivo, no solo en el propio autoconocimiento, sino, además, en el establecimiento de las relaciones interpersonales. En la investigación, en la que las variables socioemocionales y la atención plena son variables predictivas y el bienestar subjetivo es la variable criterio, participaron 117 estudiantes universitarios con parámetros de normalidad estandarizada de los cuales 34 son hombres y 83 mujeres, que siguieron de forma activa un programa de entrenamiento en Mindfulness. Los resultados obtenidos, con la aplicación de pruebas pertinentes y los procedimientos estadísticos adecuados de medidas pretest y postest, nos permiten afirmar que la competencia de atención plena y determinadas variables socioemocionales (Pensamiento Constructivo Global y Moldes de Espontaneidad Vital, Ajuste, y Optimización) correlacionan con diferencias estadísticas significativas con el bienestar subjetivo y que, además, permiten su predicción. Por otro lado, las altas puntuaciones obtenidas en el pensamiento constructivo señalan que existe la aceptación de sí mismo y hacia los demás, lo que permite mantener buenas relaciones interpersonales y actuar con coherencia y asertividad sin emitir juicos de valor.

Palabras clave: felicidad; bienestar subjetivo; Mindfulness; variables socioemocionales; pensamiento constructivo; relaciones interpersonales 


\title{
VARIABLES SOCIOEMOCIONALES Y DE ATENCIÓN PLENA EN EL BIENESTAR SUBJETIVO
}

\begin{abstract}
Socioemotional variables and mindfulness in happiness. In recent decades, the study, both theoretical and empirical, on the assessment made by people in relation to the satisfaction they may feel about their life has gained special interest in order to discover, prevent and promote the variables and resources that affect the ability to have a full and happy life. In this line, from the perspective of Positive Psychology, a research with two fundamental objectives was proposed. On the one hand, to find out what socioemotional profile (Mental Molds, Constructive Thinking and Emotional Intelligence) underlies people who have participated in a Mindfulness program, and if these variables allow predicting the index of subjective well-being. On the other hand, to assess the impact of participation in a Mindfulness program on subjective well-being, not only in selfknowledge, but also in the establishment of interpersonal relationships. In the research, in which socioemotional variables and mindfulness are predictive variables and subjective well-being is the criterion variable, 117 university students with standardized normality parameters participated following actively a Mindfulness training program, of which 34 were men and 83 women. The results obtained, with the application of relevant tests and the appropriate statistical procedures of pretest and posttest measures, allow us to affirm that mindfulness competence and certain socioemotional variables (Global Constructive Thinking and Molds of Vital Spontaneity, Adjustment, and Optimization) correlate with significant statistical differences with subjective well-being and that, in addition, they allow their prediction. On the other hand, the high scores obtained in constructive thinking indicate that there is acceptance of oneself and others, which allows maintaining good interpersonal relationships and acting with coherence and assertiveness without making value judgments.
\end{abstract}

Keywords: happiness; subjective well-being; mindfulness; socioemotional variables; constructive thinking; interpersonal relationships

\section{INTRODUCCIÓN}

A pesar del interés reciente por el conocimiento y la comprensión de la felicidad este tema ha sido objeto de estudio a lo largo de la historia al considerarla que es lo que da verdadero sentido a la vida y todas las acciones humanas (García, 2002, p.19).

Este sentido impulsor de la conducta origina que la psicología, aunque de forma tardía debido a su carácter impreciso, incluya este tópico en su campo de estudio. Como consecuencia de esta indefinicióny debido a su orientación esencialmente terapéutica y positivista, Ios primeros planteamientos científicos acerca del tema en el ámbito psicológicotuvieron un enfoque claramente experimental centrándose más en la importancia de los elementos tipo funcional, cuantitativos, externos, demográficos, economicistas y de índole objetivoy excluyeron de su foco de interés todos aquellos temas o tópicos en los que elementos subjetivos tuvieran un peso significativo.

Sin embargo, con el paso del tiempo y de forma progresiva, se empezaron a incluir estudios que contemplaran componentes internos abordándolos desde el punto de vista de la propia persona, desde su experiencia y valoración de todos los aspectos de su vida destacando una perspectiva positiva y no centrando la relevancia solo en los factores negativos. Así surge el concepto de bienestar subjetivo y se elaboran formulaciones generales mediante las que resaltan los principales constructos del Bienestar Subjetivo, señalando que estos influyen directamente en la sensación de felicidad y en la calidad de vida que cada persona percibe de sí misma (Seligman y Cskiszentmihalyi, 2000; Deneve, Diener y Xuared, 2013).

Por ello se empieza a minimizar el uso de recursos de investigación que estaban ligados solo a las enfermedades y,actualmente, se utilizan otras metodologías más sofisticadas que permiten medir, definir, explicar y comprender las cualidades humanas positivas en personas sanas, identificando así los factores que conforman el Bienestar Subjetivo. En esta línea de trabajo se considera a Diener como uno de los más importantes investigadores del Bienestar Subjetivo. Como resultado de muchas investigaciones y estudios empíricos propone que este bienestar tiene beneficios más allá de "sentirse bien", y que se refiere a la evaluación que la persona hace de su calidad de vida desde su propia perspectiva subjetiva, y valora sus circunstancias objetivas en función de sus metas, valores y 
cultura y aportan un mayor autocontrol, permiten que afloren conductas más prosociales y que las relaciones sociales sean de mayor calidad (Deneve, Diener y Xvared, 2013; Diener, Lucas, \&Oishi, 2018).

Otros investigadores Ryff (1989) y Ryff y Reyes (1995), incorporan, además de las variables citadas, la valoración que, mediante la atención, hacen las personas entre los elementos cognitivos y afectivos. Se establece una interacción entre lo racional y lo emocional a la hora de analizar la satisfacción con la vida, las relaciones positivas con los otros, la autonomía y control sobre su entorno, la congruencia entre expectativas y logros alcanzados y la aceptación de sí mismo, entre otras.

Por su parte, Kabath- Zin (2012) y Siegel (2010) destacan la importancia de la atención y conciencia plena, de la presencia activa y reflexiva y de la experiencia emocional, y argumentan que estas variables son eficaces en el incremento de las funciones cognitivas, en el fomento de unas relaciones interpersonales y en el bienestar subjetivo que sienten las personas cuando son capaces de reflexionar sobre sus actos, pero sin emitir juicios de valor. Es decir, que desarrollar y mantener una atención especial a la experiencia presente a lo que nos sucede, con una aceptación plena, libre de todo control y sin juicios de valor, no solamente genera un mayor autoconocimiento y facilita una mejor discriminación de nuestro propio comportamiento, sino sobre todo, influye en una mayor sensación de satisfacción con lo que se hace, en el bienestar personal, en la manera en que vivimos nuestra vida y en cómo nos relacionamos con los demás.

Además que el ser consciente de algo y el "darnos cuenta" de nuestros procesos de pensamiento y de nuestras acciones nos asegura el éxito y reporta grandes beneficios al desarrollo y al aprendizaje. En palabras de Simón (2015) sería esta actitud de observación, propia de la atención plena, la única maniobra que libera a la conciencia de la servidumbre de la emoción. Asimismo, Redorta (2021) resalta que "la toma de conciencia respecto de algo supone la capacidad de medir las consecuencias de lo que está sucediendo" (p. 57).

En la misma línea que los autores anteriores y fruto de nuestra inquietud en seguir profundizando en el estudio del bienestar personal y de la felicidad planteamos la siguiente investigación con la finalidad de analizar si los efectos o beneficios de un programa Mindfulness o de atención plena son debidos a determinados perfiles socioemocionales y si estos pueden condicionar el nivel de sensación de bienestar subjetivo (variable criterio), es decir, si existen determinadas variables socioemocionales propias del desarrollo cognitivo emocional que sean predictivas del índice de bienestar subjetivo, y si el desarrollo de la competencia de atención plena aporta mejoras al índice de bienestar subjetivo.

Con esta finalidad, se midió antes y después del tratamiento un indicador de conciencia plenay se analizaron los distintos niveles de mejora en función de sus variables independientes de partida, utilizando para ello los procedimientos estadísticos adecuados.

\section{OBJETIVOS DE LA INVESTIGACIÓN}

Conocer el nivel de normalidad de la muestra en las variables socioemocionales (Moldes Mentales, Pensamiento Constructivo e Inteligencia Emocional), la competencia de atención plena y el índice de bienestar subjetivo.

Conocer qué relación existe entre las variables socioemocionales, el índice de atención plena y bienestar subjetivo después de la aplicación de un programa basado en Mindfulness.

Conocer si es posible predecir el índice de bienestar subjetivo a partir de las variables socioemocionales y la participación en un programa de atención plena.

Analizar la relación de atención plena con el bienestar subjetivo en la optimización de las relaciones interpersonales.

\section{MUESTRA Y/O PARTICIPANTES}

Participaron 117 estudiantes que cursan sus estudios en distintos centros universitarios de Las Palmas de Gran Canaria de los cuales 34 son hombres y 83 mujeres con un rango de edad media entre los 20 y 23 años. 


\section{VARIABLES SOCIOEMOCIONALES Y DE ATENCIÓN PLENA EN EL BIENESTAR SUBJETIVO}

Cursaban especialidad de: Técnico Superior en Gestión de Alojamientos Turísticos, Gestión y Técnico Superior en administración y Finanza, Técnicos Superior en Educación Infantil y Grado en Magisterio.

\section{METODOLOGÍA Y/O INSTRUMENTOS UTILIZADOS}

\section{Diseño}

Se trata de una investigación no experimental, descriptiva de carácter cuantitativo y cualitativo. En la parte cuantitativa se trabajó con una metodología descriptiva, correlacional y cuasi-experimental de las variables socioemocionales, pensamiento constructivo y atención plena en relación con el índice de bienestar subjetivo. Es cuasi-experimental porque no se "manipulan experimentalmente las variables" que se van a tomar en consideración.

Para la investigación cualitativa utilizamos el análisis de las valoraciones de informantes clave por su experiencia obtenida durante el entrenamiento en el programa Mindfulness-ProAP. En situaciones de focus group se recogía, en una puesta en común conunaestructurapreviamente establecida, lasvaloraciones sobre la importancia que los participantesatribuían a la toma de conciencia plena y a las variables socioemocionales en su bienestar personal.

\section{Variables}

Predictivas:Estrategias cognitivo-emocionales (moldesmentales); variables de Pensamiento Constructivo; Habilidades de Inteligencia Emocional; Programa de intervención Mindfulness-ProAP.

Criterio: Índice de bienestar subjetivo: valoración cuantitativa de bienestar subjetivo.

\section{Instrumentos}

Para obtener los datos de las variables socio-emocionales y la atención plena y responder a las hipótesis planteadas en esta investigación se escogieron diferentes instrumentos de medida estandarizados que garantizaran los resultados obtenidos.

Test de estrategias cognitivo-emocionales MOLDES (Hernández-Guanir, 2010).

Inventario de Pensamiento Constructivo CTI (Epstein, 2012).

Escala de Inteligencia Emocional TMMS-24 basada en Trait Meta-Mood Scale (TMMS) de Salovey y Mayer, adaptada al castellano por Fernández-Berrocal, Extremera y Ramos (2004).

Escala "BIS-HERNÁN" Test de bienestar individual subjetivo (2000).

Programa Mindfulness-ProAP (Martín, Luján y Rodríguez-Mateo, 2015).

Documento ad hoc de narrativas experienciales en sesiones de focus group

\section{Procedimiento}

La participación en el programa Mindfulness era voluntaria, pero suponía una implicación activa del estudiante asistiendo a las sesiones presenciales y realizar la práctica establecida en cada sesión. El Ilamamiento para la inscripción al curso se realizó mediante diferentes medios: publicaciones en RRSS, información interna del centro, WhatsApp, carteles, etc.

Este estudio se desarrolló en tres fases consecutivas:

Fase inicial: búsqueda de referencias científicas relacionadas con el centro de estudio; recopilación y redacción del marco teórico-conceptual, descripción del problema, planteamiento del diseño, elaboración del programa de intervención Mindfulness-ProAP, elección de los instrumentos de medida y búsqueda de participantes.

Fase nuclear / central: en esta fase se implementó el programa Mindfulness-ProAP. Primeramente, se informó a los participantes del compromiso adquirido; instrumentos de evaluación, autorización de tratamientos de datos, práctica informal y puesta en común experiencial. La aplicación del programa Mindfulness-ProAP se desarrolló en cuatro sesiones presenciales durante cuatro semanas. La metodología aplicada era similar: realización 
de evaluaciones,desarrollo de contenidos, prácticas establecidas y puesta en común de las experiencias vividas, tanto en el aula como la práctica informal en casa y su inclusión a la vida cotidiana.

Para minimizar los efectos que pudieran ocasionar distorsión en los resultados, todas las sesiones fueron realizadas por la misma persona y con idénticas condiciones y recursos:

Fase análisis /conc/usiones: para el análisis de los resultados cuantitativos obtenidos se utilizó un programa estadístico SPSS - versión 23 en castellano.

$1^{0}$. Para conocer si el índice de bienestar subjetivo en la muestra tiene una tendencia de función de curva normal, lo que nos autoriza a aceptarla como una adecuada variable dependiente, se realizó el análisis de las puntuaciones obtenidas en la valoración del Bis - Hernán

$2^{0}$. Para conocer qué variables socioemocionales tienen mayor correlación con el índice de bienestar subjetivo se llevó a cabo un análisis de correlación entre las puntuaciones de índice de bienestar subjetivo y las variables socioemocionales y las diferentes medidas de atención plena: pretest, postest e incremento.

3ํ. Además, se realizó un análisis de correlación entre las puntuaciones de las diez dimensiones socioemocionales de las variables predictivas -estrategias cognitivo emocionales y la puntuación de atención plena finaly la variable criterio 0 índice de bienestar subjetivo.De este modo es posible conocer las unidades más pequeñas (dimensiones) que tienen una mayor correlación con el índice de bienestar subjetivo.

4ํ. También, se aplicó una ecuación de regresión quepermitiera predecir el índice de bienestar subjetivo en función de las dimensiones que están más fuertemente relacionadas.

$5^{0}$. Por último, para la evaluación cualitativa se realizó transcripción de las experiencias descritas en las situaciones de focus group, se establecieron categorías y subcategorías y se interpretaron los resultados de los análisis.

\section{RESULTADOS Y DISCUSIÓN}

\section{Investigación cuantitativa}

Nuestro principal objetivo es conocer qué variables socioemocionales y de atención plena correlacionan y predicen significativamente el índice de bienestar subjetivo en una muestra sana. Para ello iniciamos los análisis de parámetros de normalidad/patología para continuar con los análisis de correlación y de regresión. Por limitación de espacio en este artículo solamente destacaremos los resultados más relevantes obtenidos 


\section{VARIABLES SOCIOEMOCIONALES Y DE ATENCIÓN PLENA EN EL BIENESTAR SUBJETIVO}

Tabla 1.

Frecuencia de BIS-Hernán en los participantes.

Bis-total

\begin{tabular}{|c|c|c|c|c|c|}
\hline & & Frecuencia & Porcentaje & Porcentaje válido & $\begin{array}{l}\text { Porcentaje } \\
\text { acumulado }\end{array}$ \\
\hline \multirow[t]{23}{*}{ Válidos } & 24 & 1 & 9 & ,9 & ,9 \\
\hline & 26 & 1 & ,9 & ,9 & 1,8 \\
\hline & 27 & 1 & ,9 & ,9 & 2,6 \\
\hline & 28 & 1 & 9 & ,9 & 3,5 \\
\hline & 29 & 3 & 2,6 & 2,6 & 6,1 \\
\hline & 30 & 1 & ,9 & ,9 & 7,0 \\
\hline & 31 & 3 & 2,6 & 2,6 & 9,6 \\
\hline & 32 & 4 & 3,4 & 3,5 & 13,2 \\
\hline & 33 & 6 & 5,1 & 5,3 & 18,4 \\
\hline & 34 & 4 & 3,4 & 3,5 & 21,9 \\
\hline & 35 & 10 & 8,5 & 8,8 & 30,7 \\
\hline & 36 & 8 & 6,8 & 7,0 & 37,7 \\
\hline & 37 & 14 & 12,0 & 12,3 & 50,0 \\
\hline & 38 & 12 & 10,3 & 10,5 & 60,5 \\
\hline & 39 & 11 & 9,4 & 9,6 & 70,2 \\
\hline & 40 & 11 & 9,4 & 9,6 & 79,8 \\
\hline & 41 & 8 & 6,8 & 7,0 & 86,8 \\
\hline & 42 & 3 & 2,6 & 2,6 & 89,5 \\
\hline & 43 & 3 & 2,6 & 2,6 & 92,1 \\
\hline & 44 & 4 & 3,4 & 3,5 & 95,6 \\
\hline & 45 & 2 & 1,7 & 1,8 & 97,4 \\
\hline & 46 & 3 & 2,6 & 2,6 & 100,0 \\
\hline & Total & 114 & 97,4 & 100,0 & \\
\hline Perdidos & Sistema & 3 & 2,6 & & \\
\hline Total & & 117 & 100,0 & & \\
\hline
\end{tabular}

Con este análisis se ha comprobado que el índice de bienestar subjetivo presente en la muestra manifiesta una tendencia de función de curva normal. Este dato es muy importante pues comenzamos el estudio desde una muestra de la poblaciónlibre de sesgos y que puede ser tomada como una referencia significativa dado los parámetros de normalidad de la prueba BIS Hernán (Hernández, 2000).

Trabajar con una muestra con parámetros de normalidad estandarizada favorece la garantía de los resultados obtenidos, pues según la aportación de Atienza y cols. (2000) las personas que manifiestan bienestar subjetivo gozan de adecuada salud mental, además de otros factores como la ausencia de estrés, depresión o ansiedad 
Tabla 2.

Resumen de las correlaciones estadísticamente positivas entre variables predictivas (Pensamiento Constructivo, Encuadres de Moldes Mentales e Inteligencia Emocional) y variable criterio (Bienestar Subjetivo)

\begin{tabular}{llc}
\hline & & Bis-total \\
\hline CTI-PCG-T & Correlación de Pearson &, $401^{* *}$ \\
& Sig. (bilateral) &, 000 \\
& $\mathrm{~N}$ & 111 \\
\hline MOL-E1-T & Correlación de Pearson &, $365^{* *}$ \\
& Sig. (bilateral) &, 000 \\
& $\mathrm{~N}$ & 109 \\
\hline MOL-E2-T & Correlación de Pearson &, $449^{* *}$ \\
& Sig. (bilateral) &, 000 \\
& $\mathrm{~N}$ & 109 \\
\hline MOL-E3-T & Correlación de Pearson &, $249^{* *}$ \\
& Sig. (bilateral) &, 009 \\
& $\mathrm{~N}$ & 109 \\
\hline APPTOTAL & Correlación de Pearson &, $324^{* * *}$ \\
& Sig. (bilateral) &, 001 \\
& $\mathrm{~N}$ & 109 \\
\hline APf total & Correlación de Pearson &, $462^{* *}$ \\
& Sig. (bilateral) &, 000 \\
& $\mathrm{~N}$ & 110 \\
\hline IE Comprensión & Correlación de Pearson &, $222^{* *}$ \\
& Sig. (bilateral) &, 019 \\
& $\mathrm{~N}$ & 111 \\
\hline ** La corren &
\end{tabular}

**. La correlación es significativa al nivel 0,01 (bilateral).

*. La correlación es significativa al nivel 0,05 (bilateral).

Se comprueba, tal y como se recoge en la tabla 2, que existen algunas variables socioemocionales yla variable de competencia de atención plena cuya correlación es estadísticamente significativa con el índice de bienestar subjetivo. Las puntuaciones más significativas de la competencia de atención plena son las referidas a la atención plena final, es decir aquellas puntuaciones que se realizaron después de la participación en el programa de intervención Mindfulness-ProAP en la medida de postest.

Debido a la correlación positiva y significativa entre pensamiento constructivo y el bienestar personal podemos indicar que las personas que se caracterizan por este perfil tienen una alta aceptación de sí mismas, adaptan su forma de pensar a las necesidades de las diferentes situaciones, implicándose de forma espontánea y vital, puesto que perciben la realidad de forma positiva y sin exageraciones, con una visión coherente, asertiva y constructiva tanto en las circunstancias positivas como en las negativas. Esta disposición provoca situación de bienestar.

Además, si tenemos presente las características propias del Encuadre 2 (Ajuste), que reúne las dimensiones que justifican la forma en la que una persona interactúa con la realidad podemos definir el perfil de una persona, según Hernández-Guanir (2010), como aquella que tiene "una gran disposición mental a percibir la realidad de manera positiva y sin exageraciones, conectando con los problemas, encajando las contrariedades y actuando de modo preciso, y controlando, sin basarse en atribuciones externas ante los éxitos o fracasos". Por ello, Una puntuación positiva en este enfoque predice un mayor índice de bienestar subjetivo. Esta interacción no se hace exclusiva desde un solo ámbito, sino que abarca todas las formas de actuación: cognitiva, conativa y afectiva.

Por su parte, la correlación positiva con espontaneidad vital nos indica queenlas personas que domina este encuadre son capacesdisfrutar en cada momento de sus experiencias de forma más equitativa y sin hacer unahiperreflexiónde la realidad que les impida una actuación libre y el disfrute deesta.

Por último, las altas puntuaciones en el Encuadre 3: (Optimización) nos señala a una persona que"toma las riendas" de sus actuaciones; analiza la realidad de forma constructiva,lo que le permite potenciar sus propias 


\section{VARIABLES SOCIOEMOCIONALES Y DE ATENCIÓN PLENA EN EL BIENESTAR SUBJETIVO}

habilidades y con ello tomar iniciativa y ejercer un papel activo y de mejora sobre sus propias experiencias, potenciando sudesarrollo y crecimiento personal.Estos datos coinciden con las aportaciones de Kong et al. (2017) que señalan que las personas satisfechas con su vida tienden a ser optimistas.

Igualmente, realizamos análisis de correlación entre todas las dimensiones de los encuadres 1, 2 y 3, con la finalidad de precisar y definir el perfil socioemocional de la persona. Destacan correlaciones estadísticamente significativas en: D1 (Implicación Vital), D2 (Negatividad), D3 (Discordancia), D4 (Evitación), D5 (Defensividad), D6 (Atribución externa), D7 (Operatividad), D8 (Optimización positivizante), D9 (Optimización preparatoria) y D10 (Optimización autopotenciadora) junto a las puntuaciones obtenidas en atención plena final y el índice de bienestar subjetivo.

Tabla 3

Resumen de las correlaciones estadísticamente negativas entre variables predictivas y variable criterio

\begin{tabular}{llc} 
& & $\begin{array}{c}\text { Bis-total (variable } \\
\text { criterio) }\end{array}$ \\
\hline MOL-D2-T & Correlación de Pearson &,$- 478^{* *}$ \\
& Sig. (bilateral) &, 000 \\
& $\mathrm{~N}$ & 109 \\
\hline MOL-D3-T & Correlación de Pearson &,$- 447^{* *}$ \\
& Sig. (bilateral) &, 000 \\
& $\mathrm{~N}$ & 109 \\
\hline MOL-D4-T & Correlación de Pearson &,$- 291^{* *}$ \\
& Sig. (bilateral) &, 002 \\
& $\mathrm{~N}$ & 109 \\
\hline MOL-D5-T & Correlación de Pearson &,$- 306^{* *}$ \\
& Sig. (bilateral) &, 001 \\
& $\mathrm{~N}$ & 109 \\
\hline
\end{tabular}

Una puntuación negativa en estas dimensiones se interpretar como índice de bienestar subjetivo, puesto que si existe tendencias a no realizar anticipaciones aversivas ni a mostrar desconfianza hacia los demás (D2 Negatividad); a aceptar la realidad tal y como es, pero implicándose en lo que puede mejorar (D3 Discordancia); a conectar con los problemas afrontándolos directamente, con el pensamiento, las emociones, y la acción, sin mecanismos de escape (D4 Evitación); y tendencia a aceptar los propios fallos, encajando las contrariedades y generando alternativas(D5 Defensividad), nos encontramos con una persona que se caracteriza con afrontar con realismo y consciencia sin realizar juicios hacia las circunstancias que se tiene que enfrentar ni hacia los demás y, posee un buen autocontrol de las emociones y los pensamientos. Todo esto nos permite predecir un mayor índice de bienestar subjetivo con las variables socioemocionales y la atención plena.

Para confirmar esta predicción se realizó un análisis de regresión para obtener una ecuación estructural cuyos resultados se recogen en la tabla 4. 
Tabla 4.

Variables socioemocionales con mayor probabilidad de predicción.

\begin{tabular}{|c|c|c|c|c|c|c|}
\hline \multirow{3}{*}{\multicolumn{2}{|c|}{$\overline{\text { Modelo }}$}} & \multicolumn{3}{|c|}{ Coeficientes $^{\mathrm{a}}$} & \multirow{3}{*}{$\mathrm{t}$} & \multirow{3}{*}{ Sig. } \\
\hline & & \multicolumn{2}{|c|}{$\begin{array}{l}\text { Coeficientes no } \\
\text { estandarizados }\end{array}$} & \multirow{2}{*}{$\begin{array}{c}\begin{array}{c}\text { Coef. } \\
\text { tipificados }\end{array} \\
\text { Beta } \\
\end{array}$} & & \\
\hline & & $\mathrm{B}$ & Error típ. & & & \\
\hline \multirow[t]{2}{*}{1} & $\begin{array}{l}\text { (Constant } \\
\text { e) }\end{array}$ & 29,268 & 1,610 & & 18,179 &, 000 \\
\hline & Apftotal & , 130 & ,025 & ,460 & 5,152 &, 000 \\
\hline \multirow[t]{3}{*}{2} & $\begin{array}{l}\text { (Constant } \\
\text { e) }\end{array}$ & 29,851 & 1,563 & & 19,097 &, 000 \\
\hline & Apftotal & ,088 &, 028 &, 311 & 3,126 & ,002 \\
\hline & $\begin{array}{l}\text { MOL-E2- } \\
\mathrm{T}\end{array}$ & ,043 &, 015 & ,294 & 2,954 &, 004 \\
\hline
\end{tabular}

a. Variable dependiente: Bis-total

Los resultados que muestra la tabla 4 confirman de forma estadísticamente significativa, que la variable cognitivo emocional del Encuadre E2 (Ajuste) junto a la variable de atención plena final son predictoras del mayor índice de bienestar subjetivo. Por lo tanto, las personas con una dominancia en este Encuadre 2 (Ajuste) se caracterizan por su tendencia a admitir sus errores como parte de su responsabilidad, aceptando las consecuencias y buscando soluciones más asertivas que les permiten una vida más satisfactoria. Su capacidad para aceptar la realidad, tal y como se acontece sin atribuciones externas, ni tendencia al autoengaño favorece sus relaciones interpersonales e intrapersonales. Según los resultados obtenidos, si a estas cualidades le sumamos los beneficios que aporta la práctica y el desarrollo de la atención plena que favorecen la toma de conciencia de la realidad que vive con objetividad y reaccionando de forma asertiva y eficaz, se puede afirmar que la unión de estas dos variables son elementos clave para alcanzar el mayor índice de bienestar subjetivo.

Estos resultados coinciden con la aportación deRodríguez-Fernández y Goñi-Grandmontagne (2011) que indican queen las personas con un alto bienestar subjetivo predominan las valoraciones positivas acerca de las circunstancias de su vida sin valorar los aspectos negativos.

\section{Investigación cualitativa}

El análisis e interpretación de las aportaciones realizadas por los participantes en el programa pautado y estructurado, así como la codificación temática y la categorización nos ha permitido establecer tres categorías y3 subcategorías

Los resultados de los análisis y la mayor cantidad de respuestas repetidas hacen referencia a los beneficios del entrenamiento en atención plena:

Satisfacción con la vida a nivel cognitivo: "La participación en el curso me ha permitido reflexionar más sobre las metas que me propongo en la vida", "Ahora pienso que yo también puedo aportar cosas positivas a mis vivencias", "Ahora me detengo y planifico mi forma de actuar", " Los pensamientos que desarrollo ya no son tan negativos", "Cuando se me presenta alguna dificultad ahora la analizo un poco más y me ayuda a decidir".

Satisfacción con la vida nivel afectivo: "Ahora soy capaz de controlar más mis emociones y sufro menos", "El Mindfulness me está ayudando a controlar más mi estrés y mi ansiedad", "Ahora intento adaptarme a la situación sin enfadarme tanto", "Me siento más satisfecho de cómo voy resolviendo mis problemas", "Antes no disfrutaba con las cosas buenas de mi vida, ahora me siento feliz y eufórica".

Satisfacción con la vida en:

Los aspectos personales: "Me siento más feliz porque soy consciente de mis impulsos y logro controlarlos", 


\section{VARIABLES SOCIOEMOCIONALES Y DE ATENCIÓN PLENA EN EL BIENESTAR SUBJETIVO}

"Siento que estoy más contenta conmigo", "Tenía una inclinación a pensar de forma negativa antes de realizar cualquier cosa y ahora veo también el lado positivo" "Ahora me centro más en mí que en lo que opinen o piensen los demás".

Las interacciones con los demás: "Ya no desconfío tanto en los demás y me relaciono mejor", "Antes pensaba que la culpa de todo lo que me ocurría la tenían los demás y que los demás suelen ser personas egoístas que no ayudan a resolver los problemas", "Antes no solía confiar en las personas y ahora, mi mayor autoconocimiento me hace ser más confiado", "Antes pensaba que todo era culpa de los demás", "El estar más receptiva me ha ayudado a relacionarme mejor".

El afrontamiento de situaciones: "Ahora, no me centro tanto en los aspectos negativos e intento resaltar las cosas positivas para encontrar una solución"," Cuando hay dificultades ya no me "encierro en banda", "Ahora cuando veo que me he equivocado, intento buscar una solución y no le echo la culpa a los demás".

\section{CONCLUSIONES}

Existen variables socioafectivas y cognitivo emocionales que pueden predecir el mayor beneficio de competencia en la atención plena y bienestar subjetivo después de la intervención de un programa Mindfulness.

Se confirma la existencia de las variables socioemocionales de pensamiento constructivo general, en las estrategias cognitivo emocionales en el encuadre de ajustes, optimización y espontaneidad vital y la atención plena inicial y final correlacionan de forma estadísticamente significativa con el índice de bienestar subjetivo.

Las variables sociemocionales junto a la atención plena permiten desarrollar una capacidad óptima de gestión de las dificultades y en la toma de decisiones por ende favorece el bienestar subjetivo

La práctica del Mindfulness supone un recurso notable en la búsqueda del bienestar subjetivo, pues cumple importantes funciones tales como modular el pensamiento y reducir los niveles de estrés, angustia y ansiedad, lo que contribuye al mayor disfrute y calidad de vida de las personas que deciden integrarlo en su día a día.

\section{REFERENCIAS BIBLIOGRÁFICAS}

Atienza, F., Pons, D., Balaguer, I. y García, M. (2000). Propiedades psicométricas de la escala de satisfacción con la vida en adolescentes. Psicothema, 12, pp. 314-319.

DeNeve, JE., Diener, E., Tay, L., y Xuereb, C. (2013).Las ventajas objetivas de bienestar subjetivo.En JF Helliwell, R. Layard y J. Sachs (Eds.), World informe felicidad 2013.Volumen 2. (pp. 54-79). Red Soluciones de Desarrollo Sostenible de la ONU.

Diener, E., Lucas, R. E., \&Oishi, S. (2018). Advances and open questions in thescience of subjectivewell-being. Collabra: Psychology, 4(1), 1-78. doi:10.1525/collabra.115.

Epstein, S. (2012). Manual: Inventario de pensamiento constructivo. Una medida de inteligencia emocional: TEA Ediciones.

Fernández - Berrocal, P., Extremera, N. y Ramos, N. (2004). Validity and realibility of theSpanishmodified versión of theTrail Meta-MoodScale. Psychologica IReports, 94, $751-755$.

García, M.A. (2002). El bienestar subjetivo. Subjective well-being. Escritos de Psicología, 2002, 6, 18-39

Hernández (2000). Escala "BIS-HERNÁN" Test de bienestar individual subjetivo. Tafor Publicaciones.

Hernández_Guanir, P. (2010). Moldes. Test de estrategias cognitivas-emocionales. TEA Ediciones.

Kabat-Zinn, J. (2012). Vivir con plenitud las crisis. Cómo utilizar la sabiduría del cuerpo y de la mente para afrontar el estrés, el dolor y la enfermedad. Kairós.

Kong, F., You, X., \&Zhao, J. (2017). Evaluation of the gratitudequestionnaire in a Chinesesample of adults: Factorial validity, criterion-relatedvalidity, and measurement invariance En N.Callejay T. Mason2. Escala de Bienestar Subjetivo (EBS-20 y EBS-8): Construcción y Validación. Revista Iberoamericana de Diagnóstico y Evaluación - e Avaliação Psicológica. RIDEP · №55 · Vol.2 · 185-201 · 2020

Martín, R., Luján, I. y Rodríguez-Mateo, H. (2015). Programa Mindfulness-ProAp en R. Martín, Perfil socioemo- 
cional de los que más se benefician de un programa Mindfulness. Tesis doctoral. Universidad de Las Palmas de Gran Canaria.

Redorta, J. (2021). Cómo abordar los conflictos estructurales. Almuzara

Rodríguez-Fernández, A. y Goñi-Grandmontagne,A. (2011) La estructura tridimensional del bienestar subjetivo. Anales de psicología 2011, vol. 27, no-2 (mayo), 327-332

Ryff, C.D. (1989). Happiness Is Everything, Or Is It? Exploration sonthe Meaning od Psychological Well-Being. Journal of Personality and Social Psychology, 57, $1069-1081$.

Ryff, C. D. y Reyes, L. M. (1995) The structure of psychological well-beingre visited. Journal of Personality and Social Psychology, 69, $710-727$.

Seligman, M. y Csikszentmihalyi, M. (2000). Positive Psychology. An Introdution. American Psychologist, 55, 514.

Siegel, D. J. (2010). Cerebro y Mindfulness. Paidós.

Simón, V. (2015). La compasión: el corazón del mindfulness. Editorial Sello 
